\title{
ANALYSIS AND EXPERIMENTAL RESULTS OF UNTETHERED FLIGHT OF STEREOLITHOGRAPHICALLY PRINTED MEMS MICROFLIERS
}

\author{
Syed A. Hussain ${ }^{l}$, Andrew Klitzke ${ }^{l}$, Ratul Majumdar ${ }^{l}$, and Igor Paprotny ${ }^{1 *}$ \\ ${ }^{1}$ University of Illinois at Chicago, Chicago, USA
}

\begin{abstract}
This paper presents modeling and experimental results of untethered flight of stereo-lithographically printed MEMS microfliers propelled by radiometric forces. Analysis of the physics behind radiometric microscale flight is presented, followed by detailed experimental results. The present work further enhances our understanding of the underlying principles of these microflier designs as well as demonstrates methods to build designs using 3-D stereo-lithography. This paper verifies that the perimeter and not the surface area of the flier is the key factor in determining the force experienced during flight. Despite differences in underlying physics, similar preference (perimeter over surface area) is observed in biological microscale flight.
\end{abstract}

\section{INTRODUCTION}

Microrobotics is a new field entailing the development of microscale robotic systems, i.e. robotic devices with dimensions on the order of tens or hundreds of micrometers. Due to recent advances in micro electro mechanical systems (MEMS), this last decade has seen the development of several novel microscale mobile robotic systems. These systems include devices such as electrostatically driven stress-engineered microrobots (MicroStress-Bots) [1], resonating stepping magnetic robots [2], stick-slip magnetic walkers [3], rolling magnetic walkers [4], microscrew-based swimmers [5], and biologically-propelled swimmers [6]. Despite the successful development of several mobile microrobotic systems, untethered aerial microrobots with a maximum size of one millimeter cubed have not yet been successfully developed.

While successful aerial microrobots have not been developed in the past, the concept of microscale aerial nodes is not a new idea. Microscale surveillance nodes floating in the air was proposed (but never implemented) as early as in the mid 1980's [7]. Microscale levitating gyroscopes have been implemented using magnetic [8] and electrostatic [9] forces, but these structures are constrained to reside in well-defined sites above a substrate. Radiometric levitation of microstructures has been also proposed and theoretically analyzed [10]. Recently, untethered flight of surface micromachined structures powered by radiometric forces [11, 12] have been demonstrated. These MEMS microfliers are $300 \mu \mathrm{m} \times 300 \mu \mathrm{m} \times$ $1.5 \mu \mathrm{m}$ in size and are actuated by a temperature gradient from microfabricated heaters situated underneath them. Movies of representative flight experiments for radiometric microfliers can be found here [13].

However, in order to fully investigate the physics of microscale flight, design parameters must be explored that make the use of traditional surface micromachining difficult. The microfliers discussed in this paper have been fabricated using 2-photon polymerization, allowing potentially unrestricted $3 \mathrm{D}$ geometry. The devices are lighter than their surface-micromachined counterparts, and have achieved untethered flight to a distance of several $\mathrm{mm}$, reached heights of up to a $1 \mathrm{~mm}$ above the substrate, and velocities of up to $10 \mathrm{~mm} / \mathrm{s}$. An in-depth analysis of the radiometric forces, combined with our experiments, provides evidence that the overall perimeter of the flier, rather than its surface area, is the key factor affecting its thrust. This result seems to further be corroborated by the flight surfaces of microscale insects found in nature.

Future aerial microrobots, if successfully developed, have many applications in areas such as microscale payload delivery, radar deflection, and medicinal and sensing.

\section{RADIOMETRIC MICROFLIER ACTUATION}

Radiometrically powered microscale flight is achieved by the use of thermal transpiration effect, which is a force generated by a thermal gradient in the air surrounding the microflier. The thermal transpiration effect is caused by momentum transferred from the impact of faster moving gas particles on the underside of the microflier as shown in Fig 1. If the thermal gradient is oriented in the vertical direction, the thermal force $F$ may counteract the force due to gravity, propelling the microflier upwards. Higher momentum of gas particles below the microflier is achieved by a heating the underside gas particle using a MEMS micro-heater.

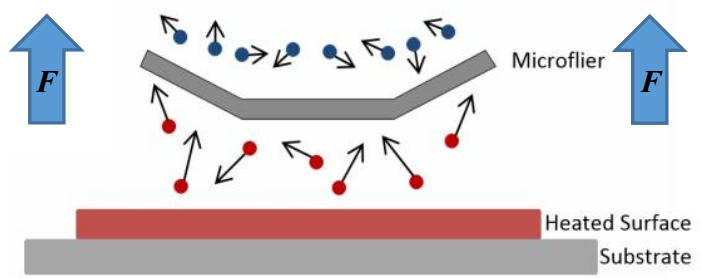

Fig. 1. The mechanism of actuation of microfliers using thermal transpiration effect.

Previous analytical treatments of the radiometric force have been performed by Einstein [14], Maxwell [15], and others. These treatments have been expanded on recently by Scandurra [16], where relationship for the net force on a thin plate in a thermal gradient was derived. In Scandurra's paper the force is divided into two components: the shear and normal components. These components are combined into a single formula (1): the force generated by the thermal transpiration effect:

$$
F_{r}=-l \cdot \Delta T \frac{(2 \alpha \lambda-\alpha \tau-4 \lambda)}{\sigma^{2} \tau} \frac{15 k}{\pi 64 \sqrt{2}} .
$$

In Eq. (1), $\alpha$ is the thermal accommodation coefficient of the surrounding air to the material, $k$ is the Boltzmann constant, $\tau$ is the thickness of the plate, $\Delta T$ is the difference in temperature between the top and bottom of the plate, $\lambda$ is the mean free path of the surrounding gas, $\sigma$ is the radius of the surrounding gas, and $l$ is the length of the perimeter around the edge of the plate. A key observation that can be made is that in (1), the force produced is directly proportional to the perimeter of the plate.

The following corollary is proposed based on Scandurra's radiometric force analysis for thin plates based in equation (1):

Corollary 1: Given two microfliers with the same horizontal crosssectional area but different perimeters, assuming all other factors remain the same, the microflier with the larger perimeter experiences greater radiometric force, i.e. $F_{r} \propto l$.

Measuring forces at the microscale is challenging. Instead of directly measuring the force, the vertical and horizontal distance travelled by the microflier can be analyzed. This is assuming that a 
higher actuation force leads to a greater vertical and horizontal travel distance. Using this, Col. 1 is extended for the horizontal distance travelled by the microflier:

$$
D_{h} \propto F_{r} \propto l
$$

where $D_{h}$ is the horizontal distance covered by the microflier. We designed microfliers and experiments to test Col 1.

\section{MICROFLIER DESIGN}

To show that the perimeter is an important factor in the radiometric force, microfliers were designed with similar horizontal cross-sections and varying perimeters. Two microfliers (Flier A and B) were designed with the perimeter of the one twice that of the other, respectively. Fig. 2 shows the CAD models of the microfliers with 4 wings (Flier A) and 16 wings (Flier B) which has twice the perimeter of 4-wing (Flier A). Other parameters for the two designs are given in Tab 1.

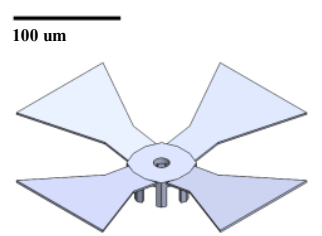

Flier A

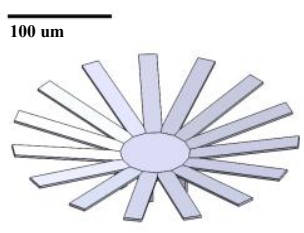

Flier B
Fig. 2. CAD models of (a) 4 winged and (b) 16-winged $3 D$ printed microfliers.

Tab. 1. Microfliers' features with horizontal surface area of 26000 $\mu m^{2}$ and $\rho$ (density) $=1370 \mathrm{~kg} / \mathrm{m}^{2}$

\begin{tabular}{|c|c|c|c|c|}
\hline Type & Wings & $\begin{array}{c}\text { Perimeter } \\
(\boldsymbol{\mu m})\end{array}$ & $\begin{array}{c}\text { Volume } \\
\left.\mathbf{( m}^{\mathbf{3}}\right)\end{array}$ & $\begin{array}{c}\text { Weight } \\
(\mathbf{n N})\end{array}$ \\
\hline Flier A & 4 & 1516 & $7.44 \mathrm{e}-14$ & 0.9988 \\
\hline Flier B & 16 & 3135 & $7.10 \mathrm{e}-14$ & 0.9532 \\
\hline
\end{tabular}

\section{FABRICATION}

Previously reported silicon microfliers used stress-engineering to angle the wings such that in-flight stability is ensured. In this work, two photon lithography is used in an additive manufacturing process to fabricate our microfliers.

Multiphoton lithography is a technique for creating small features in a photosensitive material without the use of complex optical systems or photomasks [17]. Multi-photon lithography has been successfully used in the Nanoscribe Photonic Professional GT tool for 3D microprinting and maskless lithography. Fig. 3 shows the scanning electron micrograph (SEM) of the designs for Flier A and B using stereolithography.

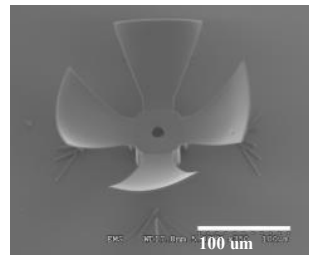

(Flier A)

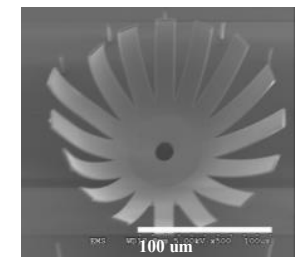

(Flier B)
Fig 3. SEM Pictures of 4 wing (Flier A) and 16 winged (Flier B) stereolithograpically printed microfliers.

Two photon absorption [18] is the simultaneous absorption of two or more photons in order to excite a molecule from a ground state to a higher excitation state. Two photon absorption has a general advantage over single-photon absorption in the fact that its resolution has a quadratic dependence on the intensity. The intensity level is just enough to polymerize the photoresist which acts as the basic material for microstructures created by this process. For creating MEMS microfliers, a stereolithography technique enables creation of a large variety of microflier geometries, enabling the evaluation of the effects of flier geometry on flight performance. This in turn enabled the investigation of the hypothesis from Col 1.

\section{EXPERIMENTAL RESULTS}

Experiments were conducted to test our hypothesis presented in Col 1. For this experiment, the 3-D printed microfliers were transferred to MEMS heaters. The heaters were fabricated from polycrystalline silicon using a surface micromaching foundry process [18] on a die with $10 \mathrm{~nm}$ of chrome metal deposited to increase the conductivity.

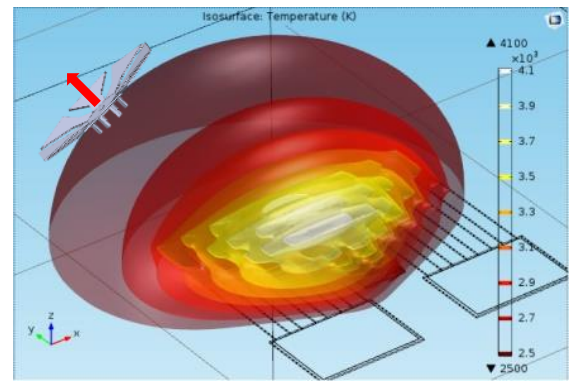

Fig. 4. Isothermal profile around the MEMS heater. Microflier flight path normal to the isothermal contour is indicated by the arrow and superimposed CAD model.

Fig 4 presents the isothermal contours around the heater as generated by FEA model. In all the experiments, the microflier is observed to fly normal to the isothermal contours.

For each experiment, a single microflier was placed at the center of a heater. A high speed optical camera (Basler Ace acA2040-180kc) connected to a microscope recorded the flight at a frame rate of 620 fps. Fig. 5 show flight sequences of a 4 wing and 16 wing microflier captured using the high speed optical camera during the activation of the heater. The location of the microflier is highlighted in red. Fig 6 shows a 16-wing microflier take-off imaged using FLIR infra-red imaging, indicating that the heater reaches a temperature of above $700{ }^{\circ} \mathrm{C}$ as the flier is propelled upwards.

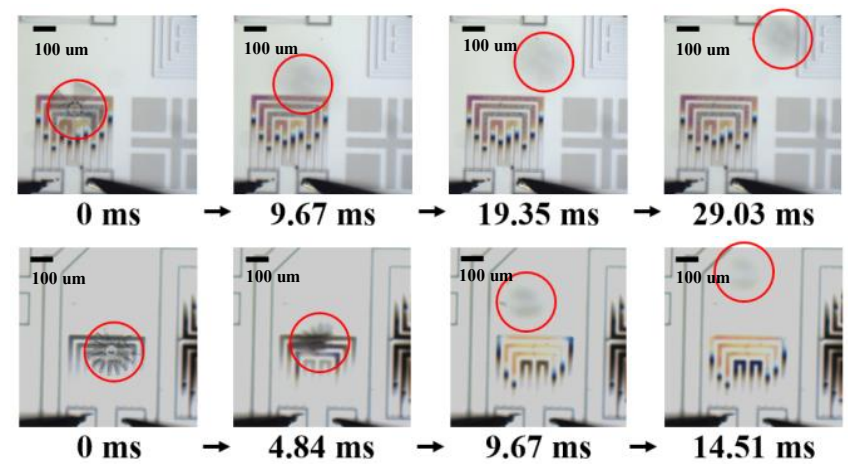

Fig. 5. Image sequences of a representative untethered flight of a 4wing (top) and 16 wing (bottom) microfliers captured by high-speed optical camera at $620 \mathrm{fps}$. 


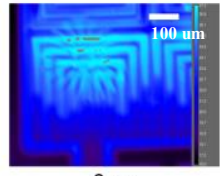

$0 \mathrm{~ms}$

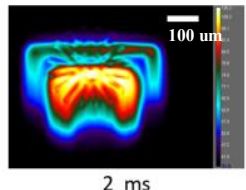

$2 \mathrm{~ms}$

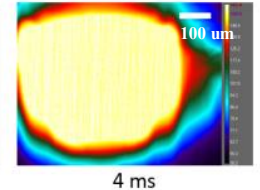

$4 \mathrm{~ms}$
Fig. 6. Take-off of $3 D$ printed microflier captured using FLIR thermal camera at $480 \mathrm{fps}$.

\section{Flight profiles}

The vertical and horizontal distance covered by the flier during flight was extracted by comparing the high-speed camera frames to a set of images taken of the microflier at known reference heights. The error to estimate the height of these flights increases with increasing height which is shown with respect to the error bar at the each point. A total of five flight profiles for each of the flier types (Flier A and B) were extracted.

Five representative flight trajectories for 4-winged (Flier A) and 16-winged (Flier B) microfliers are shown in Figs 7 and 8, respectively. The flight profiles indicate a tendency for increase in the horizontal and vertical distance traveled by the 16-winged microflier compared with the 4-winged microflier, as suggested by Col 1 .

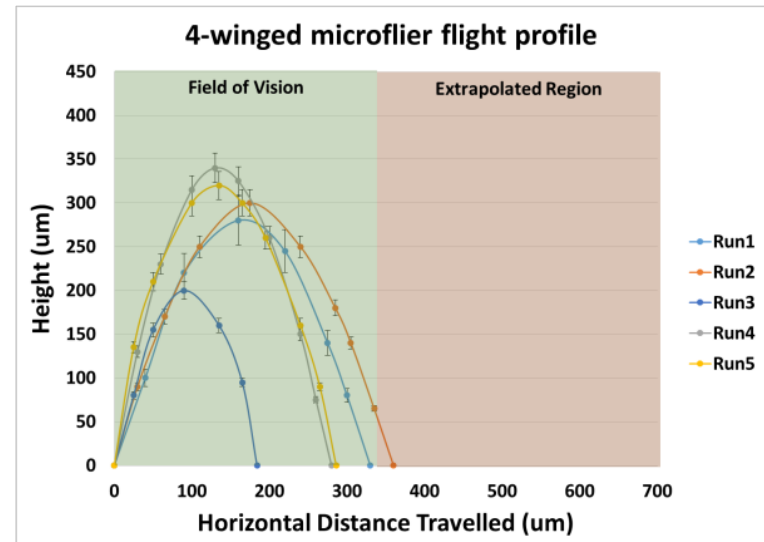

Fig 7. Flight profiles for five representative experiments with 4winged microfliers.

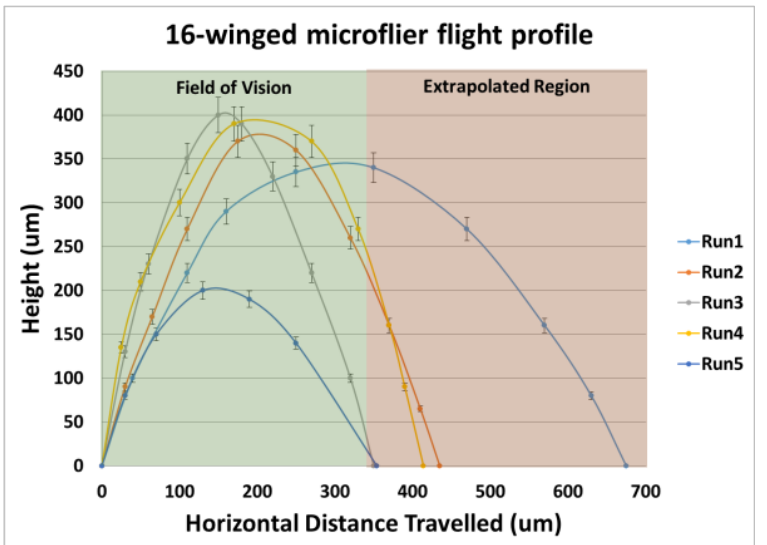

Fig. 8. Flight profiles for five representative experiments with 16winged microfliers.

According to our hypothesis, it was presumed that a larger force is experienced with a greater perimeter and hence a larger vertical or horizontal flight distance. The extracted experimental data was used to generalize our hypothesis to a larger population of microfliers.

A sampled dataset of fifteen experimental data points for each design is used to test the hypothesis. The statistical data for both microflier experiments are summarized in Tab 2.

Tab 2. Statistical analysis of the microfliers' horizontal flight distance as observed from 15 experiments for two types of microfliers.

\begin{tabular}{|c|c|c|}
\hline Statistics & $\begin{array}{c}\text { 4-Winged } \\
\text { Microflier }\end{array}$ & $\begin{array}{c}\text { 16-winged } \\
\text { Microflier }\end{array}$ \\
\hline Mean & 315.15 & 426.45 \\
\hline Standard Dev & 65.54 & 94.60 \\
\hline Confidence interval 95\% & 36.29 & 52.39 \\
\hline
\end{tabular}

Statistical t-test for fifteen samples was performed with a $95 \%$ confidence interval. Fig 9, shows the resulting 95\% confidence intervals of the data for the 4 winged and 16-winged microfliers. A paired t-test was used to reject the null hypothesis, up to the confidence of $98.25 \%$, suggesting that Flier A and B indeed fall into different distributions with respect to distance flown. It can be therefore inferred that the perimeter plays an important role in thermal flight performance of stereo-lithographically printed MEMS microfliers, confirming Col 1.

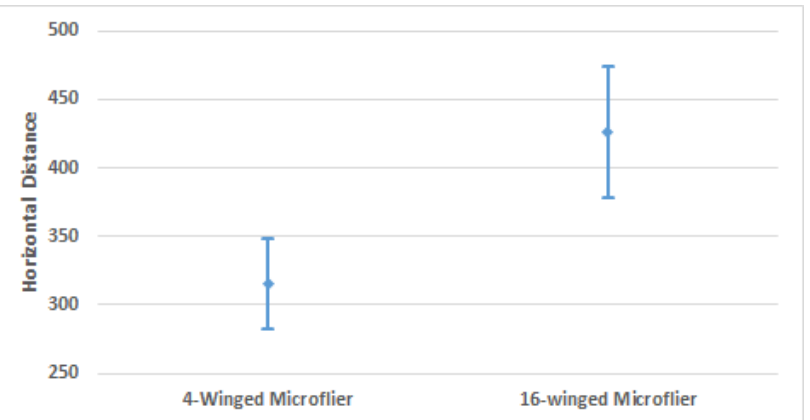

Fig. 9. 95\% confidence interval of horizontal distance travelled by two sets of 15 microfliers indicates statistically significant differences between their flight performances.

\section{Biomimetic Significance}

Although diametrically different in the physical underpinnings of the forces experienced by our radiometrically powered microfliers to the forces experienced by microscopic flying insects, a convergence to the same underlying geometry can be observed. Fig. 10 shows the body and wing designs of a recently discovered parasitic wasp [19]. The unusual wing structure consisting of fringes (setae) favoring large perimeter, can be observed. A simple analysis of laminar flow across the wings' surface at varying scales (ranging from micrometer to $\mathrm{cm}$ sized) suggest that the flow in between the gaps of these wings is very small relative to the flow outside of the gaps at the micrometer scales and at low Reynolds numbers. As the relative scale is increased, more flow is observed in-between the fringes.

Although it is unclear at this point the exact convergence between the microflier geometries and the wing geometries of the microflying insects, despite the differences in the underlying physical mechanisms, perhaps the similarities in the shear molecular flow across both sides of the wings is an explanation as to this biomimetic coincidence, warranting further investigation. 


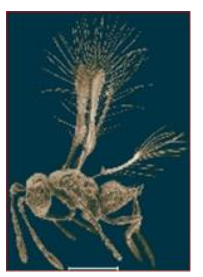

(a)

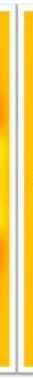

FEM Laminar flow simulation for model scaled up by factor 60

Fig. 10. (top) (a) Tinkerbella Nana parasitic wasp (b) 2D FEM model of the fringes, approximately based on cross section as shown (bottom) Simplified 2D Finite Element Model laminar flow of parasitic wasp wing fringes at different scales. Arrow indicates the air-flow.

\section{CONCLUSIONS}

This paper presents an analysis of the underlying forces that govern radiometrically-powered MEMS microfliers, develops designs based on this understanding using 3-D stereo-lithography, and presents experimental flight profiles extracted using high-speed microscopy. In particular, two microflier designs were tested experimentally and analysis of the results corroborate the theory that the perimeter of the microflier as opposed to the horizontal cross section area is the key factor determining the radiometric forces experienced by the microfliers.

The results indicate similar preference to what is found in nature with respect to flight surfaces of microscale insects. This is surprising, as microscale insects use a different underlying mechanism to generate lift, however it is possible that microfluidic behavior involving gaseous flow at the microscale leads to the emergence of this common feature, warranting further investigation of this phenomenon.

\section{AKNOWLEGDEMENTS}

This paper is partially supported by the college of engineering at the University of Illinois at Chicago, as well as grant from the National Science Foundation, award \#2016-02750. Travel support has been generously provided by the Transducer Research Foundation.

\section{REFERENCES}

[1] B. R. Donald, C. G. Levey, C. McGray, I. Paprotny, and D. Rus, "An untethered, electrostatic, globally-controllable MEMS micro-robot," Journal of Microelectromechanical Systems, 15, (2006), pp. 1-15.

[2] D. R. Frutiger, K. Vollmers, B. E. Kratochvil, and B. J. Nelson, "Small, fast, and under control: wireless resonant magnetic micro-agents," The International Journal of Robotics Research, 29(5), (2010), pp. 613-636.

[3] W. Jing, N. Pagano, and D. Cappelleri, "A novel micro-scale magnetic tumbling microrobot," Journal of Micro-Bio
Robotics, 8, (2013), pp. 1-12.

[4] W. Jing, X. Chen, S. Lyttle, Z. Fu, Y. Shi, and D. J. Cappelleri, "Design of a micro-scale magnetostrictive asymmetric thin film bimorph ( $\mu \mathrm{mab})$ microrobot," in ASME 2010 International Mechanical Engineering Congress and Exposition, American Society of Mechanical Engineers, (2010), pp. 599-607.

[5] T. Qiu, U. Choudhury, H.-H. Jeong, D. Schamel, and P. Fischer, "Bioinspired wireless microrobots in fluids," in Proceedings of the 2013 IEEE International Conference on Robotics and Automation (ICRA2013), (2013).

[6] D. Kim, A. Liu, E. Diller, and M. Sitti, "Chemotactic steering of bacteria propelled microbeads," Biomedical Microdevices, 14, (2012), pp. 1009-1017.

[7] J. M. Kahn, R. H. Katz, and K. S. Pister, "Emerging challenges: Mobile networking for smart dust," Communications and Networks, Journal of, 2(3), (2000), pp. 188-196.

[8] C. Shearwood, K. Ho, C. Williams, and H. Gong, "Development of a levitated micromotor for application as a gyroscope," sensors and actuators A: physical, 83(1), (2000) pp. 85-92.

[9] B. Damrongsak and M. Kraft, "A micromachined electrostatically suspended gyroscope with digital force feedback," in Sensors, 2005 IEEE, (2000), pp. 4-pp.

[10] B. Gotsmann, M. Despont, and U. Duerig, "Controlled thermophoresis as an actuation mechanism for noncantilevered mems devices," Microelectromechanical Systems, Journal of, 17(6), (2008), pp. 1287-1293.

[11] V. Foroutan, R. Majumdar, O. Mahdavipour, S. P. Ward, and I. Paprotny, "Levitation of untethered stress-engineered microfliers using thermophoretic (knudsen) force," in Technical Digest of the Hilton Head Workshop 2014: A SolidState Sensors, Actuators and Microsystems Workshop, pp. 105-106, (2014).

[12] S. Ward, V. Foroutan, R. Majumdar, O. Mahdavipour, S. Hussain, and I. Paprotny, "Towards microscale flight: Fabrication, stability analysis, and initial flight experiments for $300 \mu \mathrm{m} \times, 300 \mu \mathrm{m} \times 1.5 \mu \mathrm{m}$ sized untethered mems microfliers," NanoBioscience, IEEE Transactions on, 14, (2015), pp. 323331.

[13] I. Paprotny, "Micromechatronic systems laboratory (MSL)." available http://wwwl.ece.uic.edu/ paprotny/MSL_index.html, downloaded on 11/5/2015., 2015.

[14] A. Einstein, "Zur theorie der radiometerkrafte," Zeitschrift Fuer Physik, 27(1), (1924), pp. 1-6.

[15] J.C. Maxwell, "On stresses in rarifed gases arising from inequalities of temperature." Phil. Trans. R. Soc. Lond. 170, 231-256, 1879

[16] M. Scandurra, I. Fabrizio, and C. Paolo, "Gas kinetic forces on thin plates in the presence of thermal gradients." Physical Review E, 75(2), 2007.

[17] Goeppert-Mayer M (1931). "Über Elementarakte mit zwei Quantensprüngen". Annals of Physics, Vol 9 (3), 273-95.

[18] D. Koester, A. Cowen, R. Mahadevan, M. Stonefield, and B. Hardy, PolyMUMPs Design Handbook, A MUMPs Process, 10th ed. Grenoble, France: MEMSCAP, 2003.

[19] J. Huber, and J. Noyes, "A new genus and species of fairyfly, Tinkerbella nana (Hymenoptera, Mymaridae), with comments on its sister genus Kikiki, and discussion on small size limits in arthropods," J. Hymenopt. Res., 44, (2013) pp. 17-44.

\section{CONTACT}

*Igor Paprotny, Tel: +1-802-683-9081; paprotny@uic.edu. 